\title{
INTERVENCIÓN VÍA TELESALUD BASADA EN EL MODELO DENVER DE ATENCIÓN TEMPRANA PARA NIÑOS PEQUEÑOS CON AUTISMO
}

\author{
EARLY START DENVER MODEL-BASED TELEHEALTH INTERVENTION FOR YOUNG \\ CHILDREN WITH AUTISM
}

\section{Diego Pablo González Lagos ${ }^{1}$}

\begin{abstract}
RESUMEN
ElModelo Denver de Atención Temprana (ESDM) es una práctica basada en la evidencia diseñada para niños pequeños entre 12 y 48 meses de edad con alto riesgo o diagnóstico de Trastorno del Espectro Autista. Este artículo tiene como objetivo presentar el contenido y la estructura de La intervención vía telesalud basada en ESDM, según las autoras del modelo e investigaciones relacionadas utilizando el enfoque original, como un formato para la atención de Terapia Ocupacional y otras disciplinas vinculadas al área. La intervención vía telesalud basada en ESDM tiene la intención de enseñar a los padres cómo usar estrategias de temas específicos para enfocarse en múltiples habilidades en diferentes áreas del desarrollo del niño pequeño y cómo implementarlas en contexto natural dentro de las actividades de la vida diaria y de juego. La intervención de ESDM es compatible con el marco conceptual y práctico de la Terapia Ocupacional, además, asegura la calidad y efectividad de la intervención al ser una práctica basada en la evidencia. La telesalud permite llegar a un mayor número de familias con recursos comunitarios limitados y hace que las adaptaciones de la intervención sean más adecuadas para los estilos de vida y las rutinas de las familias, lo que resulta en un aprendizaje más activo y significativo. Este formato más que ser considerado como una práctica particular por el contexto de salud, debiese consolidarse como una alternativa de intervención válida y accesible para la comunidad.
\end{abstract}

\section{PALABRAS CLAVE}

Telesalud, terapia ocupacional, atención temprana, trastorno del espectro autista.

1 Licenciado en Terapia Ocupacional, Universidad de La Frontera. Terapeuta Ocupacional, Universidad de La Frontera ORCID: https://orcid. org/0000-0002-2528-9010. ESDM Certified Therapist, UC Davis MIND Institute. Institut Global d'Atenció Integral del Neurodesenvolupament (IGAIN) Correo electrónico: dgonzalez@igain.cat Correo electrónico: todiegogl@gmail.com Teléfono: +56977668049 


\section{ABSTRACT}

The Early Start Denver Model (ESDM) is an evidence-based practice designed for young children between 12 and 48 months of age with high risk ordiagnosis of Autism Spectrum Disorder. This article aims to present the content and structure of ESDM-based telehealth intervention, according to the authors of the model and research related to the original approach, as a format for Occupational Therapy and other related disciplines. The ESDM-based telehealth intervention intends to teach parents how to use topic-specific strategies to target multiple skills in different areas of young child's development and how to implement them in a natural context within daily living and play activities. The ESDM intervention is compatible with the conceptual and practical framework of Occupational Therapy, in addition, it ensures the quality and effectiveness of the intervention as it is an evidence-based practice. Telehealth allows reaching a greater number offamilies with limited community resources and makes the intervention's adaptations more suitable for the families' lifestyles and routines resulting in more active and meaningful learning. This format rather than being considered as a particular practice due to the health context, it should be consolidated as a valid and accessible alternative of intervention for the community.

\section{KEYWORDS}

Telehealth, occupational therapy, early intervention, autism spectrum disorder.

Recibido; 18/01/2021

Aceptado: 08/06/2021 


\section{INTRODUCCIÓN}

El Trastorno del Espectro Autista (TEA) o autismo, es un trastorno del neurodesarrollo, cuyas manifestaciones clínicas son evidentes desde antes del primer año de vida y se encuentran presentes a lo largo de todo el ciclo vital. Los síntomas centrales del TEA se enmarcan en deficiencias persistentes en la comunicación e interacción social en diversos contextos y en patrones restrictivos y repetitivos de comportamientos, intereses o actividades (American Psychiatric Association, 2014).

La prevalencia del TEA ha aumentado en las últimas décadas. En Chile no existe un registro actualizado, sin embargo, se utilizan los datos estadísticos internacionales para proyectar la prevalencia en el país (Ministerio de Salud, 2011). La última estimación mundial indica que 1 de cada 160 niños en el mundo presenta este diagnóstico (Elsabbagh et al., 2012). En Estados Unidos, el último estudio indica una prevalencia de 1 cada 54 niños (Maenner et al., 2O2O).

No existe un tratamiento curativo para el autismo. El tratamiento farmacológico se indica principalmente para tratar síntomas asociados al TEA, ya que no se ha establecido la eficacia para el tratamiento de los síntomas centrales (DeFilippis \& Wagner, 2016).

La tendencia actual en las intervenciones tempranas para los TEA son las Intervenciones Naturalistas Conductuales y del Desarrollo (NDBI), que integran enfoques de desarrollo y basados en relaciones con estrategias de análisis conductual aplicado (Schreibman et al., 2015). El énfasis en los NDBI es establecer rutinas de actividades conjuntas en un entorno natural, incluyendo juegos y actividades de la vida diaria para incorporar muchas oportunidades de aprendizaje.

El último reporte de prácticas basadas en la evidencia para niños, jóvenes y adultos jóvenes con autismo, muestra que las técnicas de enseñanza conductuales y las intervenciones naturalistas son las más respaldadas empíricamente (Steinbrenne et al., 2020).

El Modelo Denver de Atención Temprana (ESDM) es una NDBı diseñada para niños pequeños con autismo, de edades comprendidas entre los 12 y los 48 meses de edad. Utiliza los conocimientos existentes acerca del desarrollo típico del niño para facilitar una trayectoria de desarrollo similar en los niños pequeños con tEA (Rogers \& Dawson, 2010).

Con capacitación y certificación a través de UC Davis MIND Institute o supervisión directa de un Terapeuta Certificado, ESDM puede ser impartido por una variedad de PROFESIONALES de la atención temprana (terapia ocupacional, psicología, fonoaudiología, psicopedagogía, educación diferencial, entre otras), en centros de atención o en los propios hogares de los niños y sus familias (UC Davis MIND Institute, 2019).

La enseñanza del ESDM está integrada dentro de actividades de juego y actividades de la vida diaria y aborda distintos ámbitos del desarrollo (comunicación expresiva, comunicación receptiva, atención conjunta, imitación, habilidades sociales, juego, cognición, motricidad gruesa, motricidad fina y autocuidado). Utiliza prácticas y procedimientos didácticos combinados a partir de modelos de intervención tradicionales, tanto conductuales como basados en el desarrollo (Rogers \& Dawson, 2010).

Diversos estudios indican que el ESDM es efectivo para aumentar las capacidades cognitivas y lingüísticas de los niños, además de la interacción social y la iniciativa, y también para disminuir la gravedad de los síntomas del TEA y mejorar la conducta y las habilidades adaptativas en general (Rogers \& Dawson, 2010).

El ESDM ha tenido éxito como intervención intensiva 1:1, realizado en el hogar y con 20 horas o más por semana, impartido por profesionales de la intervención cuidadosamente supervisados y que estaban preparados para impartir el ESDM con altos niveles de seguimiento (Dawson et al., 2010).

Se han realizado investigaciones sobre los resultados de intervenciones basadas en ESDM de baja intensidad en algunos países. En Taiwán, niños que recibieron intervención de ESDM por 8 horas semanales dentro del Sistema Público de Salud, mostraron mejoras significativas en el lenguaje y el funcionamiento cognitivo general y redujeron la gravedad de los síntomas en la comunicación y el juego (Lin et al., 2020). En Austria, niños que recibieron intervención de ESDM por 4,6 horas semanales en un entorno comunitario, mostraron una tendencia hacia un mejor lenguaje receptivo y una reducción en los síntomas centrales del autismo 
informados por los padres en relación con el grupo de control (Holzinger et al., 2019). En Italia, niños que recibieron intervención de ESDM por 3 horas semanales dentro del Sistema Público de Salud, mostraron mejoras significativas en los dominios cognitivo y del lenguaje (Devescovi et al., 2016).

La práctica, teoría e investigación han enfatizado la importancia y la eficacia de las intervenciones realizadas por los padres para los niños con dificultades de desarrollo en la etapa temprana de la vida (Wallace y Rogers, 2010). Bajo esa premisa, ESDM desarrolla un pograma de entrenamiento a padres o cuidadores: el Parent-Delivered Early Start Denver Model (P-ESDM; Rogers, Dawson \& Vismara, 2012), el cual ha reportado mayor adquisición por parte de los padres en habilidades de interacción, mayores avances en el desarrollo y reducción de los síntomas centrales del autismo en los niños, y disminución en el estrés parental (Rogers et al., 2012; Estes et al., 2013; Rogers et al, 2019; Abouzeid et al, 2020). Sin embargo, trabajar con las familias en sus hogares, puede ser difícil de organizar debido a las demandas diarias, horarios familiares (escuela, trabajo, hermanos, vida familiar, etc.) y costos asociados (Goodwin, 2008). En consideración a eso, P-ESDM fue testeado en modalidad telesalud (Vismara et al., 2012; Vismara et al., 2016).

En la situación de pandemia provocada por el brote de la enfermedad por CoviD-19, las atenciones presenciales son un potencial un riesgo de contagio y carecen de continuidad (Colegio Médico de Chile, 2020). Además, el uso de elementos de protección personal podría interferir significativamente en la relación e interacción terapeuta-niño/a.

La telesalud utiliza tecnologías de la comunicación para brindar servicios especializados en tiempo real a una distancia geográfica (Dudding, 2009; Turner, 2003). Se puede acceder a ella en cualquier momento del día, en cualquier lugar con equipo básico y económico, y utiliza funciones interactivas y personalizadas para comunicarse y compartir información (Baggett et al., 2010). En el escenario actual (y en adelante), permite realizar intervenciones personalizadas, sin riesgo de contagio, con continuidad en el tiempo e independiente de barreras geográficas, costos y tiempos asociados.

La telepráctica basada en ESDM, está orientada a trasladar estrategias concretas basadas en la evidencia a la familias de niños pequeños con autismo, no con el afán de convertirlos en terapeutas de sus hijos/as, sino para optimizar al máximo las oportunidades de comunicación e interacción propias de las actividades cotidianas y de juego en sus rutinas diarias y crear una dinámica de enseñanza-aprendizaje constante.

A continuación se presenta de manera general la metodología y estructura de la telepráctica de basada en ESDM, según las autoras del modelo e investigaciones relacionadas utilizando el enfoque original. Los contenidos de la intervención se describen según el manual para profesionales en español: Modelo Denver de atención temprana para niños pequeños con autismo (Rogers \& Dawson, 2017) y del manual para padres en español: Atención temprana para su niño o niña con autismo (Rogers, Dawson, \& Vismara, 2018). La estructura de la telepráctica se sustenta desde investigaciones previamente realizadas sobre P-ESDM presencial (Rogers et al, 2012; Estes et al, 2013; Rogers et al, 2019; Abouzeid et al., 2020) y sobre P-ESDM vía telesalud (Vismara et al., 2012; Vismara et al., 2016).

\section{CONTENIDOS DE LA INTERVENCIÓN}

Las sesiones de teleterapia, al igual que el entrenamiento presencial, tienen como objetivo enseñar a los padres cómo usar 10 tópicos para apuntar a múltiples habilidades en diferentes áreas del desarrollo dentro de cualquier actividad en particular (Vismara et al., 2012; Vismara et al., 2016). Estos temas son: atención y motivación, rutinas sociales sensoriales, rutinas de actividades conjuntas, comunicación no verbal, imitación, atención conjunta, desarrollo del habla, habilidades de juego funcional y simbólico y las técnicas de enseñanza y las contingencias de aprendizaje del análisis conductual aplicado (Rogers, Dawson, \& Vismara, 2012; Rogers et al, 2012; Estes et al, 2013; Rogers et al, 2019; Abouzeid et al., 2020).

\section{Tópico 1. Ponerse bajo el foco: Atraer la atención del niño}

Objetivo: Aumentar la atención del niño hacia los padres.

Estrategias: Identificar el centro de atención del niño y situarse dentro de ese centro de atención, enfrente de él y a su altura. Eliminar (en lo posible) estímulos 
distractores que pueda competir por la atención del niño. Buscar la zona de confort social del niño y no salir de ella. Seguir la iniciativa del niño: utilizar técnicas de escucha activa, narración, ayuda e imitación (Rogers, Dawson, \& Vismara, 2018).

\section{Tópico 2. Buscar la sonrisa: Divertirse con las rutinas sociales sensoriales}

Objetivo: Aumentar el disfrute del niño durante los juegos sociales y las canciones.

Estrategias: Permanecer en el centro de atención, cara a cara con el niño y buscar la sonrisa. Crear rutinas divertidas con canciones, juegos y contacto físico, y acompañarlas con sonidos, caras y voces animadas. Utilizar objetos estimulantes para iniciar rutinas sociales sensoriales. Introducir variaciones en las rutinas cuando se vuelvan repetitivas. Hacer pausas y crear oportunidades de comunicación (Rogers, Dawson, \& Vismara, 2018).

\section{Tópico 3. Crear interacciones ida y vuelta}

Objetivo: Enseñar al niño la estructura de ida y vuelta de las actividades conjuntas.

Estrategias: Situarse y poner los materiales importantes entre el adulto y el niño e intencionar intercambios. Mantenerse en el centro de atención. Narrar, nominar y utilizar palabras sencillas, hacer canciones y efectos de sonido. Utilizar para el juego y las actividades una estructura de iniciación/preparación, tema, variación y cierre/transición. Mantener un estilo de turnos, ida y vuelta, en cada una de las cuatro partes de las actividades conjuntas (Rogers, Dawson, \& Vismara, 2018).

\section{Tópico 4. El cuerpo habla: La importancia de la comunicación no verbal.}

Objetivo: Proporcionar al niño la posibilidad de expresar deseos, sentimientos e intereses mediante el lenguaje corporal, así como enseñarle a que comprenda el lenguaje corporal de los demás.

Estrategias: Hacer pausas durante la interacción y esperar un gesto, una mirada y/o una vocalización. Añadir gestos y expresiones faciales a los juegos y a las actividades cotidianas. Dividir los materiales para practicar los gestos de las peticiones durante el juego. Crear barreras para que el niño necesite ayuda. Señalar objetos e imágenes y esperar a que el niño siga el gesto (Rogers, Dawson, \& Vismara, 2018).

\section{Tópico 5. Aprendizaje a través de la imitación}

Objetivo: Enseñar al niño a imitar vocalizaciones, gestos y acciones motoras (con y sin objetos).

Estrategias: Utilizar pares de objetos idénticos, imitar al niño cuando juegue con objetos y esperar a que también imite. Imitar las vocalizaciones y los sonidos del niño y esperar a que también imite. Utilizar ayudas parciales para fomentar la imitación motora y/o con objetos. Utilizar canciones y rutinas sociales sensoriales para desarrollar la imitación de vocalizaciones y gestos. Mantenerse dentro del centro de atención del niño (Rogers, Dawson, \& Vismara, 2018).

\section{Tópico 6. Cómo aprenden los niños: Un poco de teoría}

Objetivo: Utilizar el formato antecedente-conductaconsecuencia para comprender y enseñar nuevas conductas al niño.

Estrategias: Observar las conductas e identificar los objetivos del niño a través de esas conductas. Reconocer los antecedentes y las consecuencias de las conductas problemáticas y de las conductas deseadas. Poner atención a los refuerzos que se proporcionan y qué conductas se refuerzan. Sustituir las conductas no deseadas por conductas de sustitución igual de sencillas y eficientes que la conducta a sustituir. Reforzar las conductas de sustitución y dejar de reforzar (extinguir) las conductas no deseadas (Rogers, Dawson, \& Vismara, 2018).

\section{Tópico 7. El triángulo de la atención conjunta}

Objetivo: Enseñar al niño a mirar un objeto y compartir su interés por el, mediante el uso de conductas de atención conjunta: dar, mostrar y señalar.

Estrategias: Enseñar al niño a entregar objetos para pedir ayuda. Enseñar a entregar algo como respuesta a 
la palabra "dame" acompañada con el gesto de mano extendida, en intercambios de objetos. Dar, mostrar y señalar objetos e imágenes para compartir comentarios y el disfrute. Utilizar el gesto de señalar constantemente, en diversos contextos y responder al gesto de señalar de forma consistente, comentando y/o entregando objetos de interés (Rogers, Dawson, \& Vismara, 2018).

\section{Tópico 8. ¡Es hora de jugar!}

Objetivo: Ampliar el repertorio de juguetes de uso funcional, variado e independiente.

Estrategias: Enseñar al niño a jugar con sus juguetes. Iniciar con los juguetes más sencillos, servir primero de modelo y después dar ayudas (gestual, verbal y/o física) si es necesario. Utilizar la estructura de cuatro etapas de las actividades conjuntas (iniciación/preparación, tema, variación y cierre/transición). Fomentar el juego independiente organizándolo bien, sentándose detrás del niño y retirándose. Rotar los juguetes para evitar el aburrimiento (Rogers, Dawson, \& Vismara, 2018).

\section{Tópico 9. Juego simbólico}

Objetivo: Enseñar al niño a desarrollar un juego simbólico espontáneo, creativo y flexible.

Estrategias: Utilizar objetos cotidianos durante el juego. Hacer que muñecos, animales y personajes cobren vida. Seguir la iniciativa del niño en las acciones de juego espontáneo. Sustituir los objetos por otras cosas que no tengan un significado o uso claro. Enseñar al niño a jugar con objetos "invisibles". Combinar varias acciones para representar escenas cotidianas. Representar interacciones sociales y otras experiencias nuevas para ayudar a comprenderlas (Rogers, Dawson, \& Vismara, 2018).

\section{Tópico 10. El lenguaje hablado}

Objetivo: Ayudar al niño a utilizary entender el lenguaje hablado.

Estrategias: Tratar los sonidos como si fueran palabras e imitar los sonidos del niño. Aumentar el número de veces que el niño oye sonidos y onomatopeyas procedentes del entorno. Asegurarse de que el niño escuche las palabras que se espera enseñar. Utilizar lenguaje sencillo, siguiendo la regla de una palabra más. Ser constante en las palabras utilizadas para garantizar una respuesta (Rogers, Dawson, \& Vismara, 2018).

\section{ESTRUCTURA DE LA INTERVENCIÓN}

\section{Sesiones iniciales o "línea base"}

El terapeuta y los padres realizan una o dos sesiones vía teleconferencia, sincrónicas, a través de computadora o smartphone, para observar las interacciones padre-hijo en contexto natural, dentro del juego, la comida y/o actividades de cuidado. Se insta a los padres a realizar una actividad como lo harían normalmente con su hijo, en la comodidad de su propio hogar. No hay instrucción o expectativa de la "manera correcta" de realizar la actividad, sino que se espera una interacción lo más natural posible para resaltar conductas positivas y/o desafiantes reales en la dinámica familiar. Los padres seleccionan los juguetes o juegos físicos preferidos del niño (por ejemplo: esconderse, perseguir, hacer cosquillas, caballito) para involucrar a su hijo en el juego, y/o tareas de cuidado (por ejemplo: alimentar, vestir, cambiar pañales, ordenar) para demostrar el nivel de independencia de los niños dentro de estas rutinas (Vismara et al., 2012; Vismara et al., 2016).

El terapeuta en conjunto con los padres completan la Lista de Verificación del Currículum del EsDM (Rogers \& Dawson, 2017), una herramienta basada en criterios de referencia y que proporciona secuencias de adquisición de habilidades del niño enen diferentes ámbitos del desarrollo: comunicación receptiva, comunicación expresiva, habilidades sociales, imitación, cognición, atención conjunta, juego, motricidad fina, motricidad gruesa, conducta e independencia personal. Al final de la evaluación, los padres en conjunto con el terapeuta, identifican las habilidades de cada ámbito que definen las las habilidades más maduras y consistentes en el niño, aquellas que se encuentran parcialmente adquiridas y las que todavía no forman parte de su repertorio. El terapeuta puede sugerir actividades, interacciones o estrategias para ayudar a los padres a evaluar habilidades específicas. En general, se identifican un conjunto de 10 a 12 habilidades (entre las que se encuentran parcialmente adquiridas) para enseñar a los niños a lo 
largo del programa de entrenamiento remoto, sujetas a reevaluación tras 12 semanas de intervención (Vismara et al., 2012; Vismara et al., 2016).

\section{Sesiones de intervención}

Se programan sesiones con frecuencia semanal, de una hora de duración cada una, a través de las cuales se trabajan en orden cada uno de los tópicos mencionados anteriormente. Al comienzo de la sesión, se revisa el progreso de los padres sobre el tópico anterior (o se introduce el primer tópico) durante los primeros 5 minutos, seguido de una actividad de juego libre entre padres e hijo de unos 5 minutos de duración, que proporciona datos sobre los comportamientos de interés de los padres y del niño. La actividad también permite al terapeuta observar el manejo de los padres en tópico de la semana anterior $y$, si es necesario, entrenar $y$ fortalecer el uso de la técnica durante actividades adicionales en el hogar antes de pasar al siguiente tema. Luego, en los siguientes 10 minutos, el terapeuta introduce el siguiente tópico (o continúa en el mismo) con comentarios de los padres y ejemplos sobre la relevancia y adecuación de las estrategias del tópico para las necesidades de aprendizaje del niño, así como los desafíos anticipados para probar las actividades en casa con su hijo. Durante los próximos 30 minutos, los padres ponen en práctica el conjunto de nuevas estrategias en al menos dos actividades en el hogar (de juego, alimentación, higiene y/o vestuario) destinadas a enseñar las habilidades de la lista de objetivos de desarrollo identificados para el niño. Entre cada actividad el terapeuta retroalimenta el desempeño de los padres y sugiere las modificaciones necesarias según los objetivos y tópicos revisados. Finalmente, en los últimos 10 minutos, se discute la generalización hacia las actividades diarias, los padres establecen metas para la semana, se selecciona el siguiente tópico y se resuelven dudas o preocupaciones (Vismara et al., 2012; Vismara et al., 2016).

\section{Discusión}

Por su esencia naturalista y el uso del juego y las actividades cotidianas como medio terapéutico, la intervención de ESDM es totalmente compatible con el marco conceptual y práctico de la Terapia Ocupacional, además, aporta un cuerpo teórico, razonamiento clínico y técnicas de intervención basados en la evidencia científica que permiten garantizar la calidad y efectividad de la intervención. La implementación de prácticas basadas en la evidencia debiese constituir un deber ético y moral del profesional terapeuta ocupacional hacia el compromiso con la calidad de vida de los usuarios y sus familias.

El formato de atención vía telesalud basada en ESDM proporciona una método estructurado, sistemático y empíricamente validado de intervención dirigida a los padres $y$ /o cuidadores de niños pequeños con autismo, brindándoles herramientas para enseñar a sus hijos/as a conectar, comunicarse y aprender a través de las actividades diarias en el hogar, siendo ellos los agentes activos en el proceso de enseñanza aprendizaje, pudiendo prescindir de la presencialidad y descentrando la intervención de la figura del terapeuta.

La telesalud no solo ayuda a los profesionales a superar las barreras sanitarias impuestas para controlar la pandemia de covid-19 en el escenario actual, sino que también permite llegar a un mayor número de familias con recursos comunitarios limitados y hace que las adaptaciones de la intervención sean adecuadas a los estilos de vida y las rutinas de cada una, lo que resulta en un aprendizaje más activo y significativo. Por lo tanto, esta modalidad no debe considerarse como una forma de práctica particular por el contexto de salud, sino que en el futuro debiese consolidarse como una alternativa válida y accesible para la comunidad.

\section{ReferenCIAS BibLIOGRÁfiCAS}

Abouzeid, N., Rivard, M., Mello, C., Mestari, Z., Boulé, M., \& Guay, C. (2020). Parent coaching intervention program based on the Early Start Denver Model for children with autism spectrum disorder: Feasibility and acceptability study. Research in Developmental Disabilities, 105, 1-13. doi:10.1016/j.ridd.2020.103747

American Psychiatric Association. (2014). Manual Diagnóstico y Estadístico de los Trastornos Mentales DSM-5 (5 ed.). Médica Panamericana.

Baggett, K. M., Davis, B., Feil, E. G., Sheeber, L. L., Landry, S. H., Carta, J. J., \& Leve, C. (2010). Technologies for expanding the reach of evidence-based interventions: preliminary results for promoting social-emotional development in early childhood. Topics in Early Childhood Special Education, 29(4), 226-238. doi:10.1177/0271121409354782

Colegio Médico de Chile. (2020). El impacto del COVID-19 en el Sistema de Salud y propuestas para la reactivación. 
Dawson, G., Rogers, S., Munson, J., Smith, M., Winter, J., Greenson, J., . .. Varley, J. (2010). Randomized, controlled trial of an intervention for toddlers with autism: the Early Start Denver Model. Pediatrics, 125(1), 17-23. doi:10.1542/peds.2009-0958

Defilippis, M., \& Wagner, K. D. (2016). Treatment of Autism Spectrum Disorder in children and adolescents. Psychopharmacology Bulletin, 18-41.

Devescovi, R., Monasta, L., Mancini, A., Bin, M., Vellante, V., Carrozi, M., \& Colombi, C. (2016). Early diagnosis and Early Start Denver Model intervention in autism spectrum disorders delivered in an Italian Public Health System service. Neuropsychiatric Disease and Treatment, 12, 1379-1384. doi:10.2147/NDT.S106850

Dudding, C. C. (2009). Digital videoconferencing: applications across the disciplines. Communication Disorders Quarterly, 30(3), 178-182. doi:10.1177/1525740108327449

Elsabbagh, M., Divan, G., Koh, Y.-J., Kim, Y. S., Kauchali, S., Marcín, C., ... Fombonne, E. (2012). Global prevalence of autism and other pervasive developmental disorders. Autism research: official journal of the International Society for Autism Research, 160-179. doi:10.1002/aur.239

Estes, A., Vismara, L., Mercado, C., Fitzpatrick, A., Elder, L., Greenson, J., ... Rogers, S. (2013). The Impact of Parent-Delivered Intervention on Parents of Very Young Children with Autism. Journal of Autism and Developmental Disorders, 44(2), 353-365. doi:10.1007/ s10803-013-1874-z

Goodwin, M. S. (2008). Enhancing and accelerating the pace of autism research and treatment. Focus on Autism and Other Developmental Disabilities, 23, 125-128. doi:10.1177/1088357608316678

Holzinger, D., Laister, D., Vivanti, G., Barbaresi, W. J., \& Fellinger, J. (2019). Feasibility and Outcomes of the Early Start Denver Model Implemented with Low Intensity in a Community Setting in Austria. Journal of Developmental \& Behavioral Pediatrics, 40(5), 354-363. doi:10.1097/DBP.0000000000000675

Lin, T. L., Chiang, C. H., Ho, S. Y., Hsin-Chi, W., \& Ching-Ching, W. (2020). Preliminary clinical outcomes of a short-term low-intensity Early Start Denver Model implemented in the Taiwanese public health system. Autism, 1-7. doi:10.1177/1362361319897179

Maenner, M. J., Shaw, K. A., Baio, J., Washington, A., Patrick, M., DiRienzo, M.,... Dietz, P. M. (2020). Prevalence of Autism Spectrum Disorder Among Children Aged 8 Years - Autism and Developmental Disabilities Monitoring Network, 11 Sites, United States, 2016. MMWR Surveillance Summaries, 69(4), 1-12. doi:10.15585/mmwr.ss6904al

Ministerio de Salud. (2011). Guía de práctica clínica de detección y diagnóstico oportuno de los Trastornos del Espectro Autista (TEA). Santiago: Ministerio de Salud.

Rogers, S., \& Dawson, G. (2010). The Early Start Denver Model for Young Children with Autism: Promoting Language, Learning, and Engangement. New York: Guilford Press.
Rogers, S., \& Dawson, G. (2017). Modelo Denver de atención temprana para niños pequeños con autismo: Estimulación del lenguaje, el aprendizaje y la motivación social. Autismo Ávila.

Rogers, S., Dawson, G., \& Vismara, L. (2012). An early start for your child with autism: using everyday activities to help kids connect, communicate and learn. New York: Guilford Press.

Rogers, S., Dawson, G., \& Vismara, L. (2018). Atención temprana para su niño o niña con autismo: Cómo utilizar las actividades cotidianas para enseñar a los niños a conectar, comunicarsey aprender. Autismo Ávila.

Rogers, S., Estes, A., Lord, C., Vismara, L., Winter, J., Fitzpatrick, A., ... Dawson, G. (2012). Effects of a Brief Early Start Denver Model (ESDM)-Based Parent Intervention on Toddlers at Risk for Autism Spectrum Disorders: A Randomized Controlled Trial. Journal of the American Academy of Child \& Adolescent Psychiatry, 57(10), 1052-1065. doi:10.1016/j.jaac.2012.08.003

Rogers, S., Estes, A., Vismara, L., Munson, J., Zierhut, C., Greenson, J., ... Talbott, M. (2019). Enhancing Low-Intensity Coaching in Parent Implemented Early Start Denver Model Intervention for Early Autism: A Randomized Comparison Treatment Trial. Journal of Autism and Developmental Disorders, 49(2), 632-646. doi:10.1007/ s10803-018-3740-5

Schreibman, L., Dawson, G., Stahmer, A. C., Landa, R., Rogers, S., McGee, G. G., ... Halladay, A. (2015). Naturalistic developmental behavioral interventions: Empirically validated treatments for autism spectrum disorder. Journal of Autism and Developmental Disorders, 45(8), 2411-2428. doi:10.1007/s10803-015-2407-8

Steinbrenner, J., Hume, K., Odom, S., Morin, K., Nowell, S., Tomaszewski, B., . . Savage, M. (2020). Evidence-Based Practices for Children, Youth, and Young Adults with Autism. The University of North Carolina at Chapel Hill, Frank Porter Graham Child Development Institute, National Clearinghouse on Autism Evidence and Practice Review Team.

Turner, J. W. (2003). "Telemedicine: expanding health care into virtual environments. En T. L. Thompson, A. D. Dorsey, K. I. Miller, \& R. Parrott, Handbook of Health Communication (págs. 141-161). USA: Lawrence Erlbaum Associates.

UC Davis MIND Institute. (30 de Julio de 2019). Policy Statement-Using the ESDM in Community Settings. Obtenido de Early Start Denver Model: https://www.esdm.co/

Vismara, L. A., McCormick, C. E., Wagner, A. L., Monlux, K., Nadhan, A., \& Young, G. S. (2016). Telehealth parent training in the Early Start Denver Model: Results from a randomized controlled study. Focus on Autism and Other Developmental Disabilities, 33(2), 1-13. doi:10.1177/1088357616651064

Vismara, L. A., Young, G. S., \& Rogers, S. J. (2012). Telehealth for Expanding the Reach of Early Autism Training to Parents. Autism Research and Treatment, 2012, 1-12. doi:10.1155/2012/121878

Wallace, K. S., \& Rogers, S. J. (2010). Intervening in Infancy: Implications for Autism Spectrum Disorders. Journal of Child Psychology and Psychiatry, 52(5), 1300-1320. doi:10.1111/j.1469-7610.2010.02308.x 